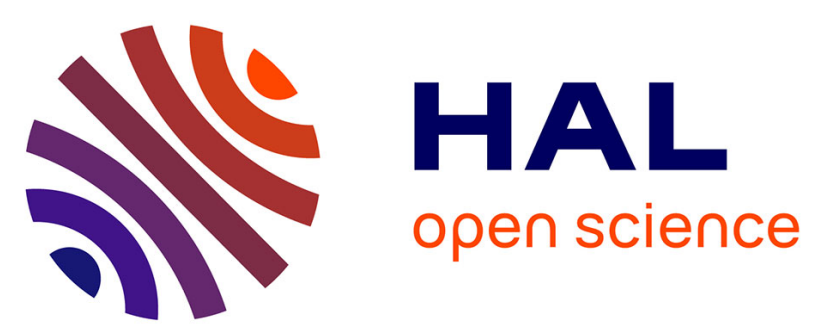

\title{
Processive Movement of MreB-Associated Cell Wall Biosynthetic Complexes in Bacteria
}

Julia Dominguez Escobar Dominguez Escobar, Arnaud A. Chastanet, Alvaro H. A. H. Crevenna, Vincent V. Fromion, Roland R. Wedlich-Söldner, Rut R. Carballido y Lopez

\section{To cite this version:}

Julia Dominguez Escobar Dominguez Escobar, Arnaud A. Chastanet, Alvaro H. A. H. Crevenna, Vincent V. Fromion, Roland R. Wedlich-Söldner, et al.. Processive Movement of MreB-Associated Cell Wall Biosynthetic Complexes in Bacteria. Science, 2011, 333 (6039), pp.225-2258. 10.1126/science.1203466 . hal-01000382

\section{HAL Id: hal-01000382 https://hal.science/hal-01000382}

Submitted on 29 May 2020

HAL is a multi-disciplinary open access archive for the deposit and dissemination of scientific research documents, whether they are published or not. The documents may come from teaching and research institutions in France or abroad, or from public or private research centers.
L'archive ouverte pluridisciplinaire HAL, est destinée au dépôt et à la diffusion de documents scientifiques de niveau recherche, publiés ou non, émanant des établissements d'enseignement et de recherche français ou étrangers, des laboratoires publics ou privés. 


\section{Processive Movement of MreB-Associated Cell Wall Biosynthetic Complexes in Bacteria \\ Julia Domínguez-Escobar et al. \\ Science 333, 225 (2011);}

DOI: $10.1126 /$ science. 1203466

This copy is for your personal, non-commercial use only.

If you wish to distribute this article to others, you can order high-quality copies for your colleagues, clients, or customers by clicking here.

Permission to republish or repurpose articles or portions of articles can be obtained by following the guidelines here.

The following resources related to this article are available online at www.sciencemag.org (this information is current as of January 4, 2013 ):

Updated information and services, including high-resolution figures, can be found in the online version of this article at:

http://www.sciencemag.org/content/333/6039/225.full.html

Supporting Online Material can be found at:

http://www.sciencemag.org/content/suppl/2011/06/01/science.1203466.DC1.html

A list of selected additional articles on the Science Web sites related to this article can be found at:

http://www.sciencemag.org/content/333/6039/225.full.html\#related

This article cites 36 articles, 15 of which can be accessed free:

http://www.sciencemag.org/content/333/6039/225.full.html\#ref-list-1

This article has been cited by 10 articles hosted by HighWire Press; see:

http://www.sciencemag.org/content/333/6039/225.full.html\#related-urls

This article appears in the following subject collections:

Cell Biology

http://www.sciencemag.org/cgi/collection/cell_biol 
lighted examples of proximal opposing movements (Fig. 4, E and F; fig. S15; and movies S13, A to D). Thus, PGEM and MreB filaments move in both directions around the cell, and we could not resolve any coordination along the cell length.

It thus appears that the coupled motions of the PGEM and MreB reflect the active process of cell wall synthesis: a circumferential motion of disconnected MreB-PGEM complexes moving around the cell in both directions, synthesizing discrete radial bands of PG oriented perpendicular to the cell length (fig. S16). This model is consistent with the arrangement of $B$. subtilis $\mathrm{PG}$ observed by atomic force microscopy (19).

MreB filaments are required for elongationspecific PG synthesis $(2,20)$, suggesting that they are integral components of these translocating machines. These filaments may serve as coordinating scaffolds to link the PGEM to the enzymes that synthesize PG precursors $(1-4,10)$ (fig. S17). We cannot completely rule out the contribution of polymer dynamics to these motions, because there are no methods to inhibit MreB polymerization without disrupting existing filaments. However, we did not observe any directed motion in the absence of PG synthesis, even with high-precision measurements (fig. S13), suggesting that PG synthesis is the predominant process driving these motions. If these motions are driven by MreB polymerization or another process, this would require induction of equivalent rigor states during depletions of all PGEM components and the antibiotic inhibition of PG cross-linking, polymerization, and precursor synthesis.

Rather than a contiguous helical structure, these observations reveal the mobile, fragmented nature of MreB. Thus, although MreB is required for rod-shape maintenance, it cannot function as a cell-spanning structure, much less a coherent "cytoskeleton" in B. subtilis. It remains to be determined how the short-range activities of these independent biosynthetic complexes impart a long-range order to the cell wall.

\section{References and Notes}

1. T. Kruse, ]. Bork-Jensen, K. Gerdes, Mol. Microbiol. $\mathbf{5 5}$ 78 (2005).

2. Y. Kawai, K. Asai, J. Errington, Mol. Microbiol. 73, 719 (2009).

3. F. O. Bendezú, C. A. Hale, T. G. Bernhardt, P. A. de Boer, EMBO J. 28, 193 (2009).

4. C. L. White, A. Kitich, ]. W. Gober, Mol. Microbiol. 76, 616 (2010).

5. J. A. Mayer, K. J. Amann, Cell Motil. Cytoskeleton 66, 109 (2009).

6. F. van den Ent, L. A. Amos, J. Löwe, Nature 413, 39 (2001).

7. T. Kruse, J. Møller-Jensen, A. Løbner-Olesen, K. Gerdes, EMBO J. 22, 5283 (2003).

8. L. J. Jones, R. Carballido-López, J. Errington, Cell 104, 913 (2001).

9. Z. Gitai, N. A. Dye, A. Reisenauer, M. Wachi, L. Shapiro, Cell 120, 329 (2005).

10. R. M. Figge, A. V. Divakaruni, J. W. Gober, Mol. Microbiol. 51, 1321 (2004).

11. R. A. Daniel, J. Errington, Cell 113, 767 (2003).

12. R. Carballido-López, Microbiol. Mol. Biol. Rev. 70, 888 (2006).
13. R. Carballido-López, ]. Errington, Dev. Cell 4, 19 (2003).

14. H. J. Soufo, P. L. Graumann, Curr. Biol. 13, 1916 (2003).

15. S. Y. Kim, Z. Gitai, A. Kinkhabwala, L. Shapiro, W. E. Moerner, Proc. Natl. Acad. Sci. U.S.A. 103, 10929 (2006).

16. H. J. Defeu Soufo, P. L. Graumann, Mol. Microbiol. 62, 1340 (2006).

17. See supporting material on Science Online.

18. R. E. Thompson, D. R. Larson, W. W. Webb, Biophys. J. 82, 2775 (2002)

19. E. J. Hayhurst, L. Kailas, ]. K. Hobbs, S. J. Foster, Proc. Natl. Acad. Sci. U.S.A. 105, 14603 (2008).

20. T. Uehara, J. T. Park, J. Bacteriol. 190, 3914 (2008).

Acknowledgments: We thank P. Graumman, J. Errington,

R. Carballido-Lopez, D. Kearns, D. Popham, and

D. Scheffers for strains; D. Kahne, S. Walker,

T. Bernhardt, and T. Uehara for discussions; Q. Justman for editing; S. Layer for inspiration; T. Emonet for MicrobeTracker; and the HMS Nikon Imaging Center for the use of their facilites. Funding was provided by NIH grants R01-GM073831 (D.Z.R.), R01-GM096450 (X.Z.), and R01-GM39565 (T.M.). X.Z. is a Howard Hughes Medical Investigator. E.C.G. was supported by the Helen Hay Whitney Foundation.

\section{Supporting Online Material}

www.sciencemag.org/cgi/content/full/science.1203285/DC1 Materials and Methods

SOM Text

Figs. S1 to S17

Tables $\mathrm{S} 1$ to $\mathrm{S} 5$

Movies S1 to S13

References

25 January 2011; accepted 20 May 2011

Published online 2 June 2011;

10.1126/science. 1203285

\section{Processive Movement of} MreB-Associated Cell Wall Biosynthetic Complexes in Bacteria

Julia Domínguez-Escobar, ${ }_{1}^{1}$ Arnaud Chastanet, ${ }^{2,3 *}$ Alvaro H. Crevenna, ${ }^{1 *}$ Vincent Fromion, ${ }^{4}$ Roland Wedlich-Söldner, ${ }^{1} \dagger$ Rut Carballido-López ${ }^{2,3} \dagger$

The peptidoglycan cell wall and the actin-like MreB cytoskeleton are major determinants of cell shape in rod-shaped bacteria. The prevailing model postulates that helical, membrane-associated MreB filaments organize elongation-specific peptidoglycan-synthesizing complexes along sidewalls. We used total internal reflection fluorescence microscopy to visualize the dynamic relation between MreB isoforms and cell wall synthesis in live Bacillus subtilis cells. During exponential growth, MreB proteins did not form helical structures. Instead, together with other morphogenetic factors, they assembled into discrete patches that moved processively along peripheral tracks perpendicular to the cell axis. Patch motility was largely powered by cell wall synthesis, and MreB polymers restricted diffusion of patch components in the membrane and oriented patch motion.

$\mathrm{T}$ he peptidoglycan (PG) layer, or sacculus, which is composed of glycan strands crosslinked by peptide bridges, forms a loadbearing network that maintains bacterial cell shape. Synthesis and chemical composition of PG are well understood (1), but the structure of the sacculus and the mechanisms controlling its growth remain elusive. It is currently assumed that the actin-like MreB proteins form filamentous helical structures along the membrane, which direct cell wall growth by positioning multienzyme complexes that mediate sidewall elongation (2). These elongation complexes are thought to contain the essential transmembrane proteins MreC and MreD, RodA and RodZ, PG hydrolases, and penicillin-binding proteins (PBPs), the enzymes that catalyze $\mathrm{PG}$ elongation and cross-linking $(3,4)$. Studies using fluorescently labeled vancomycin (Van-FL) have revealed helical incorporation patterns of $\mathrm{PG}$ precursors into the sidewall in B. subtilis (5) and Caulobacter crescentus (6), but could not resolve the dynamics of cell wall synthesis. We used total internal reflection fluorescence microscopy (TIRFM), a sensitive method for studying events at cell surfaces $(7,8)$, to observe the dynamics of the MreB cytoskeleton and its relationship with cell wall growth.

Functional green fluorescent protein (GFP) fusions to the three MreB isoforms in B. subtilis (MreB, Mbl, and MreBH) expressed at wildtype levels (fig. S1) formed discrete patches in exponentially growing cells (Fig. 1A). Patches were restricted to the cell periphery (fig. S2A) and exhibited continuous movement along linear tracks roughly perpendicular to the long axis of the cell (Fig. 1B and movie S1). To reconcile our findings with the helical structures described for MreB proteins $(2,9)$, we simultaneously imaged cells by TIRFM and conventional epifluorescence. Owing to the increased depth of field, MreB patterns visualized by epifluorescence could be misinterpreted as "helical" (Fig. 1C). In addition, MreB localized to transverse bands as cells entered stationary phase (fig. S2B). We found

${ }^{1}$ Max Planck Institute of Biochemistry, Am Klopferspitz 18, D-82152 Martinsried, Germany. ${ }^{2}$ INRA, UMR1319 Micalis, F-78352 Jouy-en-Josas, France. ${ }^{3}$ AgroParisTech, UMR Micalis, F-78350 Jouy-en-Josas, France. ${ }^{4}$ INRA, UR1077 Mathématique, Informatique et Génomes, F-78352 Jouy-en-Josas, France.

*These authors contributed equally to this work. †To whom correspondence should be addressed. E-mail: wedlich@biochem.mpg.de (R.W.-S.); rut.carballido-lopez@jouy. inra.fr (R.C.-L.) 
D

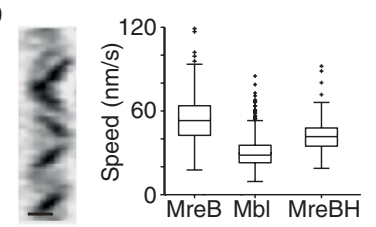

$\mathbf{F}$

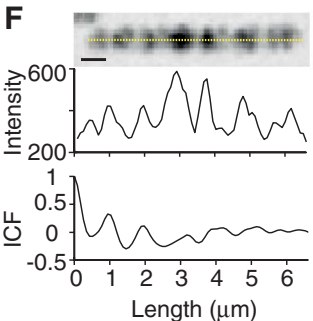

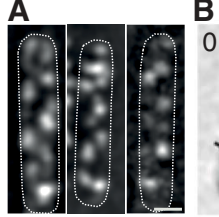

C

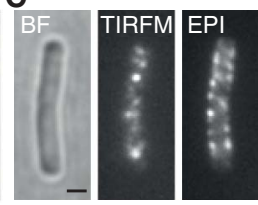

E

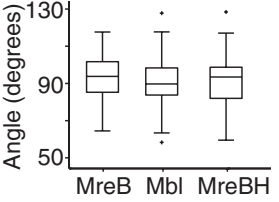

G

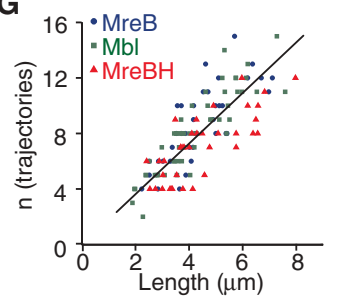

Fig. 1. Motile cell wall elongation complexes. (A) GFP-MreB, GFP-Mbl, and GFP-MreBH (left to right) imaged by TIRFM. Data in (A) to (G) are from strains 3723, 2523, and 2566] (table S4). (B) Movement of a GFP-MreB patch (arrow) and trajectory in maximum projection. Time is in seconds. (C) Simultaneous visualization of GFP-Mbl by TIRFM and epifluorescence (EPI). (Left) Corresponding bright-field (BF) image. (D) Typical kymograph and patch speed boxplots of MreB isoforms. (E) Typical maximum projection and angle boxplots for MreB isoform trajectories. (F) Distribution of GFPMreB trajectories in maximum projection, linescan (along dotted line) and intensity correlation function (ICF). (G) Number of patch trajectories
H
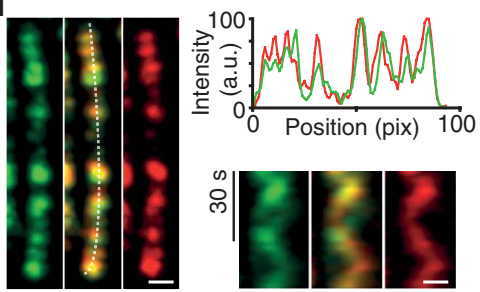

1

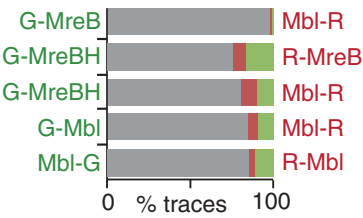

J

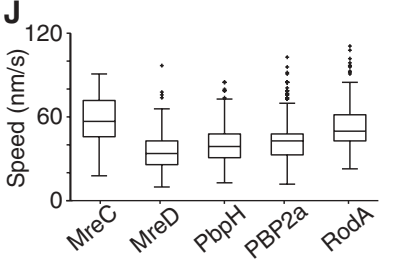

K

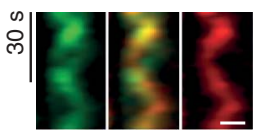

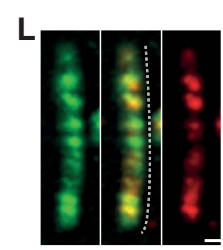

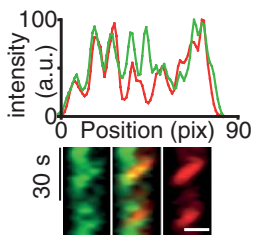

increases with cell length (linear fit: $R^{2}=0.61$ ). (H) Colocalization of GFP-MreB and Mbl-RFP in maximum projections, linescan (dotted line) and kymographs. (I) Quantification of MreBs colocalization from kymograph traces. G: GFP; R: RFP; gray bars: colocalization; green and red bars: single GFP and RFP color traces. (J) Boxplots of patch speed of morphogenetic proteins. (K) Orientation of patch trajectories of morphogenetic proteins and Van-FL bands. (L) Colocalization of GFP-PbpH and RFP-MreB in maximum projections, linescan, and kymograph traces. (M) Quantification of MreB and Mbl colocalization with PbpH and RodA from kymograph traces. Labels as in (I). Scale bars: $1 \mu \mathrm{m}$. similar patch-like localization and dynamics of MreB in the Gram-negative bacteria Escherichia coli and $C$. crescentus (fig. S2C), suggesting that these are widely conserved features in bacteria.

Detailed analysis of patch dynamics using kymographs (10) showed that patches moved bidirectionally across the cell at constant velocity (fig. S2D). We often observed reversal and crossover of patches (fig. S2D), but did not see abrupt patch appearances or disappearances at midtrajectory, indicating tight association of MreBs with the cell periphery. To determine patch velocities, we measured the slopes of kymograph traces. Patches formed by MreB, Mbl, and MreBH moved at significantly different speeds (Fig. 1D, fig. S3, and table $\mathrm{S} 1 ; P<0.001$ for all pairs). Speeds were mildly dependent on growth temperature (fig. S4A) but were not affected by high concentrations of magnesium (fig. S4B) or untagged endogenous copies of the respective MreB isoforms (fig. S4C and table S1). Patch trajectories in maximum projections were oriented at angles close to $90^{\circ}$ relative to the long axis of the cell (Fig. 1E and table S1). Although tracks were evenly spaced along the length of the cell, with major distance peaks between 0.5 and $1 \mu \mathrm{m}$ (Fig. 1F), high variability of autocorrelation between cells (fig. S5, A to C) and over time (fig. S5D) argued against a constrained periodic structure. However, we did find a correlation between numbers of tracks and

Fig. 2. Patch motility is not driven by treadmilling. (A) Time series and kymograph showing fission and fusion (arrowhead) and reversal of a GFP-Mbl patch (asterisk, RWSB10). Red: TIRFM; green: epifluorescence; blue (cell outline): bright field. (B) Time series and kymograph (dotted line) showing splitting of a GFPMreB patch (RWSB1). (C) TIRF-FRAP with partial photobleaching of a moving GFPMreB patch (RWSB1). (D) Kymographs along patch traces of GFP-Mbl in the $\Delta m r e b \Delta m b l$ background (RWSB10), with corresponding intensity profiles showing lack of fluorescence recovery upon partial bleaching. (E) Inverse FRAP (iFRAP). A cell (RWSB10) was bleached within the region outlined, omitting only a GFP-Mbl patch. Kymograph (dotted line) shows

A

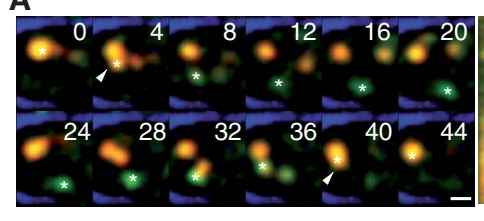

C

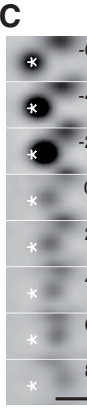

D

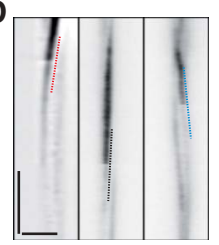

E
B
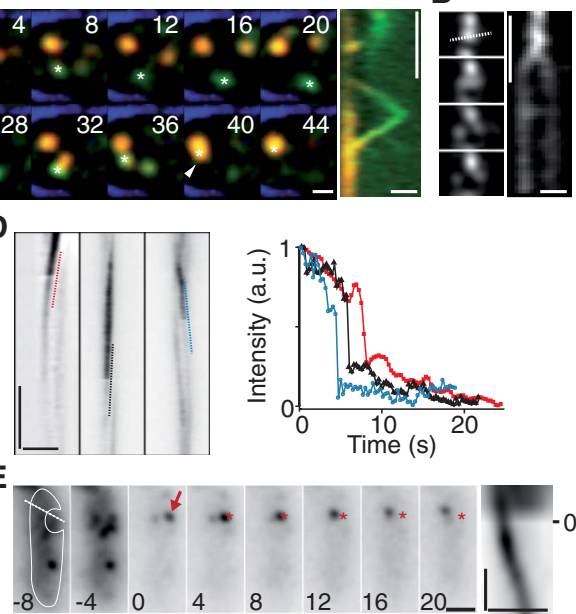

movement of the patch with no loss of fluorescence. Scale bars: $1 \mu \mathrm{m}$; time bars: $30 \mathrm{~s}$ in (A) and (B); $10 \mathrm{~s}$ in (D) and (E). Time is in seconds. Asterisks and arrow at initial position in (C) and (E).

cell length (Fig. 1G), suggesting an average distance between tracks of $\sim 0.5 \mu \mathrm{m}$, as previously reported (11).

$\mathrm{MreB}, \mathrm{Mbl}$, and $\mathrm{MreBH}$ have been reported to colocalize and interact extensively with each other $(12,13)$. To determine whether differences in patch speeds (Fig. 1D) reflected distinct isoform composition or simply different strain backgrounds, we performed two-color TIRFM on pairs of GFP- and mRFPruby- (monomeric RFP, red fluorescent protein) tagged proteins. All tested pairs displayed extensive overlap (Fig. 1H) with colocalization in more than $75 \%$ of kymograph traces (Fig. 1I and table S3) and moved at similar speeds (fig. S6 and table S1), indicating that the three MreB isoforms coexist in motile patches.

MreB and Mbl have been implicated in the spatial organization of lateral cell wall synthetic 
Fig. 3. Contribution of PG synthesis to patch motility. (A and B) Immobile MreB, Mbl, and $\mathrm{PbpH}$ patches after treatment with vancomycin $(100 \mu \mathrm{g} / \mathrm{ml})$ for $8 \mathrm{~min}(A)$ and with phosphomycin $(700 \mu \mathrm{g} / \mathrm{ml})$ for $30 \mathrm{~min}$ (B). Time is in seconds. (C and D) Effects of vancomycin (C) and phosphomycin (D) are reversible. Kymographs were taken before $(-)$ and immediately after addition of the drug (0) and at the indicated times (in minutes) after washout. Red arrows: partial recovery of motility. (E) Kymographs of GFP-Mbl patches (2523) showing partial arrest (a), complete arrest (b), or diffusive motility (c) after treatment with lysozyme $(1 \mu \mathrm{g} / \mathrm{ml})$ for $5 \mathrm{~min}$. (F and $\mathbf{G})$ Patch speed boxplots upon low phosphomycin $(1$ to $5 \mu \mathrm{g} / \mathrm{ml})$ treatment relative to untreated cells (F) and in the presence and absence of $p b p H$ or $p b p A$ (G). Scale bars: $1 \mu \mathrm{m}$; time bars: $30 \mathrm{~s}$.

Fig. 4. Roles of MreBs in patch motility. (A and B) Boxplots of patch speeds (A) and trajectory angles (B) in the presence and absence of mreB. (C) Movement of a GFP-Mbl patch (asterisk) along the cell axis in the $\Delta m r e b \Delta m b l$ strain (RWSB10). Red: TIRFM; green: epifluorescence; blue (cell outline): bright field. Time is in seconds. (D) Normalized PbpH and RodA patch motility (patch trajectories per kymograph trace per minute) after deletion of $m r e B$ or $\mathrm{mbl}$. (E) Typical kymographs for $\mathrm{PbpH}$ and RodA patches in wildtype and $m r e B$ backgrounds. Scale bars: $1 \mu \mathrm{m}$; time bar: $30 \mathrm{~s}$.
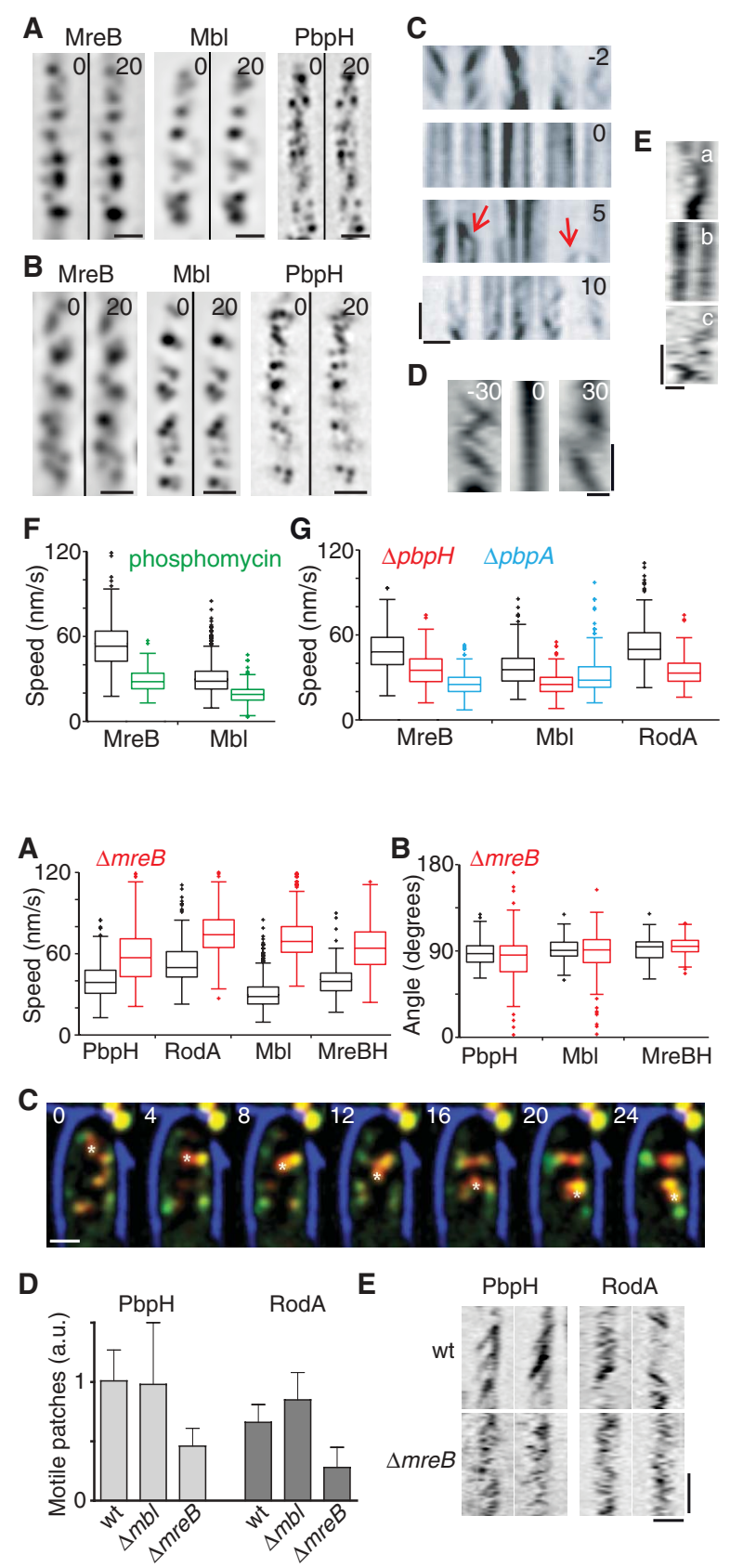

colocalized with MreB and Mbl (Fig. 1, L and M, fig. S6, and table S1). Thus, MreBs, MreC/D, RodA, and $\mathrm{PbpH} / 2 \mathrm{a}$ form part of cell wall elongation complexes that display circumferential processive motility in $B$. subtilis cells.

What is the molecular basis for the processive movement? One possibility would be treadmilling of MreB, as previously suggested in B. subtilis (11) and C. crescentus (19). However, several dynamic behaviors of MreB patches such as fusion and fission (Fig. 2A and movie S4), reversal of direction (Fig. 2A), or splitting (Fig. 2B) argue against treadmilling-driven motion. To directly test for turnover of MreBs within patches, we performed FRAP (fluorescence recovery after photobleaching) experiments. When individual GFP-MreB or GFP-Mbl patches were partially bleached, their motility was unaffected and no fluorescence recovery occurred during their movement across the cell (Fig. 2, C and D). Similarly, when a whole cell was bleached, with the exception of a single GFP-Mbl patch (Fig. 2E), the signal from this patch did not decrease during its movement across the cell, again ruling out treadmilling-driven motion. Absence of treadmilling does not, however, preclude polymerization and slow global turnover of MreB (20), which is predicted from the correlation between cell length and patch numbers (Fig. 1F).

Alternatively, a motive force for MreB patches, and thus for the elongation complexes, could be provided by PG synthesis itself. To test this possibility, we treated cells with vancomycin $(100 \mu \mathrm{g} / \mathrm{ml})$ to inhibit incorporation of PG precursors into the sacculus (21), or phosphomycin $(700 \mu \mathrm{g} / \mathrm{ml})$, which blocks the first cytosolic step in PG precursor synthesis (22). Addition of either antibiotic completely stopped movement of $\mathrm{PbpH}$, MreB, and $\mathrm{Mbl}$ patches (Fig. 3, A and B). After removal of the drugs, motility gradually resumed (Fig. 3, C and D, and movie S5). Finally, upon disruption of the PG backbone with lysozyme, patch movement also ceased (Fig. 3E and movie S6), and many patches rapidly diffused in the cytosol (Fig. 3E and movie S6). If PG assembly directly drives motility of MreB patches, the concentration of PG precursors available should influence patch speed. Reducing the precursor pool with low concentrations (1 to $5 \mu \mathrm{g} / \mathrm{ml}$ ) of phosphomycin did not affect the direction of MreB and Mbl patches (fig. S9A), but their velocities were significantly lower (Fig. $3 \mathrm{~F}$ and table S1). FRAP experiments showed no turnover of MreB or Mbl patches in the presence of either high or low concentrations of phosphomycin (fig. S8), confirming the absence of MreB turnover in patches. To target PG synthesis genetically without inducing drastic changes in cell morphology, we analyzed MreB, Mbl, and RodA patches in mutants null for $p b p H$ and pbpA (encodes PBP2a), which display a wild-type growth rate and morphology during vegetative growth $(17,23)$. Both deletions significantly slowed down MreB and Mbl patches (Fig. 3G), suggesting a role for transpeptidases in setting complexes $(4,14)$. We analyzed the localization of proteins associated with sidewall elongation by TIRFM. MreC and MreD, transmembrane and to couple the cytosolic MreBs to the extracellular PG synthetic machinery $(4,15)$, formed discrete patches that behaved like MreB patches in speed and orientation (Fig. 1, J and K, table (10), and movie S2). In B. subtilis, several PBPs sidewall in distinct foci and bands. Of the 11 vegetatively expressed PBPs of $B$. subtilis, only the transpeptidase $\mathrm{PbpH}$ formed patches that moved around the cell periphery (Fig. 1, J and $\mathrm{K}$, table S1, and movie S3). All other PBPs and LytE localized to patches that randomly moved along the cell surface (fig. S7A). In cells lacking
pbpH, PBP2a patches displayed directional the proposed reciprocal redundancy of these two which has been linked to cell wall elongation and to $\mathrm{PbpH}$ and PBP2a $(17,18)$, also formed circumially moving patches (Fig. 1, J table S1, and movie S3). Finally, the orientation of Van-FL-labeled tracks was also centered around $90^{\circ} \mathrm{C}$ (Fig. 1K) instead of helical (5). Whereas MreBs and $\mathrm{MreC}$ patches exclusively displayed circumferential motion, MreD, PBP2a, PbpH, and RodA patches also frequently exhibited rapid diffusion along the membrane (fig. S7B). Circumferentially motile $\mathrm{PbpH}$ and RodA strongly 
the pace of new PG strand assembly. Indeed, the essential transpeptidase PBP2 of $E$. coli plays a direct role in glycan strand synthesis apart from its role in cross-linking (24). Again, track orientation was unaffected (fig. S9B). Mutants in $p b p H$ and $p b p A$ were slightly impaired in growth (fig. S9C), consistent with a link between patch motility and cell growth.

Our results indicate that cell wall synthesis itself provides the driving force for the processive motility of sidewall elongation complexes. This is also supported by recent analysis showing that PG glycosyl transferases are processive enzymes (25). What, then, is the biological function of the essential actin-like MreB? MreB positions cytosolic enzymes involved in $\mathrm{PG}$ precursor synthesis in $C$. crescentus $(6,14)$. Thus, MreB patches might simply act as passive scaffolds for synthesis and export of PG precursors and/or regulate their delivery to sites of active PG synthesis (24). Our observation that RodA and PBPs localize not only to MreB-associated motile elongation complexes but also to rapidly diffusing patches (fig. S7A) suggested an alternative scenario, in which MreB polymers might actively restrict and/or control the mobility of cell wall elongation complexes. To test this hypothesis, we monitored patch motility in the absence of individual MreB isoforms. Patches moved much faster in the $m r e B$ mutant, whereas deletion of $m b l$ or $m r e B H$ had no significant effect (Fig. 4A, fig. S10, and table S1). In addition, GFP-Mbl patches in a $\triangle m b l \Delta m r e B$ background exhibited less uniform directionality (Fig. 4B), sometimes even following trajectories along the cell axis (Fig. 4C and movie S7). An even stronger effect was observed on RodA and $\mathrm{PbpH}$ : Processively motile patches were almost completely elimi- nated, and the few remaining covered very short distances, albeit faster (Fig. 4, D and E). Thus, MreB may function directly in restricting and/or organizing motility of cell wall elongation complexes, although an additional role in the recruitment of PG precursors cannot be excluded.

We propose a model for sidewall elongation in $B$. subtilis, where motile membrane-associated elongation complexes insert new PG along bands largely perpendicular to the long cell axis. Old PG strands are used as guiding scaffolds, and motility of the complexes is powered by PG polymerization. MreB-like polymers restrict the diffusion of the complexes within the membrane to achieve processive and correctly oriented movement. A similar scenario has been proposed for plant cells, where processive motility of cellulose synthase complexes is suggested to be constrained by cortical microtubules (26).

References and Notes

1. J. V. Höltje, Microbiol. Mol. Biol. Rev. 62, 181 (1998).

2. R. Carballido-López, Microbiol. Mol. Biol. Rev. 70, 888 (2006).

3. R. Carballido-López, A. Formstone, Curr. Opin. Microbiol. 10, 611 (2007).

4. W. Margolin, Curr. Biol. 19, R812 (2009).

5. R. A. Daniel, J. Errington, Cell 113, 767 (2003).

6. A. V. Divakaruni, C. Baida, C. L. White, ]. W. Gober, Mol. Microbiol. 66, 174 (2007)

7. J. H. Yu, A. H. Crevenna, M. Bettenbühl, T. Freisinger, R. Wedlich-Söldner, J. Cell Sci. 124, 1533 (2011).

8. T. C. Fleming et al., Genes Dev. 24, 1160 (2010).

9. M. T. Cabeen, C. Jacobs-Wagner, Annu. Rev. Genet. 44, 365 (2010).

10. Materials and methods are available as supporting material on Science Online.

11. H. ]. Defeu Soufo, P. L. Graumann, EMBO Rep. 5, 789 (2004).

12. R. Carballido-López et al., Dev. Cell 11, 399 (2006).

13. H. J. Defeu Soufo, P. L. Graumann, Mol. Microbiol. 62 , 1340 (2006).
14. C. L. White, A. Kitich, J. W. Gober, Mol. Microbiol. 76 , 616 (2010)

15. D. Claessen et al., Mol. Microbiol. 68, 1029 (2008)

16. D. J. Scheffers, L. J. Jones, J. Errington, Mol. Microbiol. 51, 749 (2004)

17. Y. Wei, T. Havasy, D. C. McPherson, D. L. Popham, J. Bacteriol. 185, 4717 (2003).

18. A. O. Henriques, P. Glaser, P. J. Piggot, C. P. Moran Jr., Mol. Microbiol. 28, 235 (1998).

19. S. Y. Kim, Z. Gitai, A. Kinkhabwala, L. Shapiro, W. E. Moerner, Proc. Natl. Acad. Sci. U.S.A. 103, 10929 (2006).

20. R. Carballido-López, J. Errington, Dev. Cell 4, 19 (2003).

21. D. Kahne, C. Leimkuhler, W. Lu, C. Walsh, Chem. Rev. 105, 425 (2005)

22. E. D. Brown, E. I. Vivas, C. T. Walsh, R. Kolter, ]. Bacteriol. 177, 4194 (1995)

23. T. Murray, D. L. Popham, P. Setlow, J. Bacteriol. 179, 3021 (1997).

24. T. Uehara, J. T. Park, J. Bacteriol. 190, 3914 (2008).

25. D. Barrett et al., ]. Biol. Chem. 282, 31964 (2007).

26. A. R. Paredez, C. R. Somerville, D. W. Ehrhardt, Science 312, 1491 (2006).

Acknowledgments: We thank R. Daniel and L. J. Wu for sharing unpublished strains and for helpful suggestions, D.-]. Scheffers for strains, N. Campo for the antibody against GFP, and P. Hardy for manuscript editing. This work was supported by a Human Frontier Science Program (HFSP) Young Investigator grant (R.C.-L. and R.W.-S., HFSP-RGY0067/2009-C); a European Molecular Biology Organization short-term fellowship (R.C.-L), The French National Research Agency (ANR) (R.C.-L., ANR-08-JC]C-0024-01), and the Max Planck society (R.W.-S.).

Supporting Online Material

www.sciencemag.org/cgi/content/full/science.1203466/DC1 Materials and Methods

Figs. $\mathrm{S} 1$ to $\mathrm{S} 10$

Tables $\mathrm{S} 1$ to $\mathrm{S5}$

References (27-37)

Movies $\mathrm{S} 1$ to $\mathrm{S7}$

27 January 2011; accepted 20 May 2011

Published online 2 June 2011;

10.1126/science.1203466

\section{Phosphorylation of the Autophagy Receptor Optineurin Restricts Salmonella Growth}

Philipp Wild, ${ }^{1}$ Hesso Farhan, ${ }^{2}$ David G. McEwan, ${ }^{1}$ Sebastian Wagner, ${ }^{3}$ Vladimir V. Rogov, ${ }^{4,5}$ Nathan R. Brady, ${ }^{6}$ Benjamin Richter, ${ }^{1}$ Jelena Korac, ${ }^{7}$ Oliver Waidmann, ${ }^{1}$ Chunaram Choudhary, ${ }^{3}$ Volker Dötsch, ${ }^{4}$ Dirk Bumann, ${ }^{2}$ Ivan Dikic ${ }^{1,7 *}$

Selective autophagy can be mediated via receptor molecules that link specific cargoes to the autophagosomal membranes decorated by ubiquitin-like microtubule-associated protein light chain 3 (LC3) modifiers. Although several autophagy receptors have been identified, little is known about mechanisms controlling their functions in vivo. In this work, we found that phosphorylation of an autophagy receptor, optineurin, promoted selective autophagy of ubiquitin-coated cytosolic Salmonella enterica. The protein kinase TANK binding kinase 1 (TBK1) phosphorylated optineurin on serine-177, enhancing LC3 binding affinity and autophagic clearance of cytosolic Salmonella. Conversely, ubiquitin- or LC3-binding optineurin mutants and silencing of optineurin or TBK1 impaired Salmonella autophagy, resulting in increased intracellular bacterial proliferation. We propose that phosphorylation of autophagy receptors might be a general mechanism for regulation of cargo-selective autophagy.

M acroautophagy (hereafter referred to as autophagy) is an evolutionarily conserved catabolic process by which cells deliver bulk cytosolic components for degradation to the lysosome (1-4). Selectivity in cargo targeting is mediated via autophagy receptors that simul- taneously bind cargoes and autophagy modifiers, autophagy-related protein 8 (ATG8)/ microtubuleassociated protein light chain 3 (LC3) $/ \gamma$-aminobutyric acid receptor-associated protein (GABARAP) proteins, which are conjugated to the autophagosomal membranes $(5,6)$. The regulatory mechanisms controlling the spatiotemporal dynamics of the autophagy receptor-target interaction in cells remain unclear (7).

${ }^{1}$ Frankfurt Institute for Molecular Life Sciences and Institute of Biochemistry II, Goethe University School of Medicine, TheodorStern-Kai 7, D-60590 Frankfurt (Main), Germany. ${ }^{2}$ Infection Biology, Biozentrum, University Basel, Klingelbergstr. 50/70, CH-4056 Basel, Switzerland. ${ }^{3}$ The Novo Nordisk Foundation Center for Protein Research, Faculty of Health Sciences, University of Copenhagen, Blegdamsvej 3, 2200 Copenhagen, Denmark. ${ }^{4}$ Institute of Biophysical Chemistry and Center for Biomolecular Magnetic Resonance, Goethe University, Marie Curie Strasse 9, D-60439 Frankfurt (Main), Germany. ${ }^{5}$ Institute of Protein Research, 142290, Pushchino, Russia. ${ }^{6}$ Systems Biology of Cell Death Mechanisms, German Cancer Research Center, Bioquant, Im Neuenheimer Feld 267, 69120 Heidelberg, Germany. ${ }^{7}$ University of Split, School of Medicine, Department of Immunology and Medical Genetics, Soltanska 2, 21000 Split, Croatia.

*To whom correspondence should be addressed. E-mail: ivan.dikic@biochem2.de 